\title{
Size of Endometrioma and Number does Influence the Ovarian Reserve: A Prospective Observational Study
}

\author{
${ }^{1}$ MR Sandya, ${ }^{2}$ Pratap Kumar
}

\section{ABSTRACT}

Background: Endometriosis is one of the most commonly encountered benign problems in gynecology. Ultrasound and endocrine parameters have been widely accepted as markers of ovarian reserve. Anti-Mullerian hormone $(\mathrm{AMH})$ in conjugation with antral follicle counts is now believed to be an excellent measure for detecting ovarian reserve. Surgical approach has a fundamental role in the management of endometriosis. The loss of normal follicles can be studied by histopathological assessment of the cyst wall. The postoperative decline in the ovarian reserve is believed to have a correlation with number of endometriomas and diameter of the cyst wall.

Objective: This prospective observational study was undertaken to evaluate the effect of laparoscopic cystectomy with respect to number and size of the cyst on ovarian reserve parameters.

Materials and methods: Fifty patients undergoing laparoscopic endometrioma cystectomy were analyzed. Cysts of $<5 \mathrm{~cm}$ and $>5 \mathrm{~cm}$ and the number of cysts were studied. Statistical analysis was done using Mauchly's test of sphericity. Anti-Mullerian hormone and antral follicle count were estimated prior to and 1 month after surgery. Pre- and postoperative values were compared and analyzed with respect to number and size of endometrioma.

Observation and results: There was an overall drop of $\mathrm{AMH}$ from $3.8 \pm 3.01$ to $2.67 \pm 1.92 \mathrm{ng} / \mathrm{ml}(\mathrm{p}<0.001)$. The mean AMH in bilateral and unilateral endometrioma was $2.9 \pm 1.7$ and 3.9 $\pm 3.17 \mathrm{ng} / \mathrm{ml}$ respectively. Anti-Mullerian hormone dropped from $4.53 \pm 3.4$ to $3.19 \pm 2.18$ with $<5 \mathrm{~cm}$ cyst compared with 2.4 \pm 1.2 to $1.7 \pm 0.85$ with $>5 \mathrm{~cm}$ cyst $(p<0.01)$. Overall drop in AFC was $5.17 \pm 1.44$ and $3.61 \pm 1.61$ pre- and postoperative respectively $(p<0.01)$. Mean drop in AFC was 2.2 and 1.2 in cyst $<5$ and $>5 \mathrm{~cm}$ respectively. Histopathological analysis showed loss of follicles in $25 \%$ of the cyst walls. This was correlating with the drop in both AMH and AFC postoperatively.

Conclusion: Size and number of cyst does affect ovarian reserve to the effect that smaller and bilateral cyst leads to a greater decline in the same.

Keywords: Anti-Mullerian hormone, Antral follicle count, Cyst size, Laparoscopic endometrioma cystectomy, Number.

How to cite this article: Sandya MR, Kumar P. Size of Endometrioma and Number does Influence the Ovarian Reserve: A Prospective Observational Study. Int J Infertil Fetal Med 2016;7(1):14-18.

\footnotetext{
${ }^{1}$ Resident, ${ }^{2}$ Professor

1,2Department of Obstetrics and Gynecology, Kasturba Medical College, Manipal, Karnataka, India
}

Corresponding Author: MR Sandya, Resident, Department of Obstetrics and Gynecology, Kasturba Medical College Manipal, Karnataka, India, Phone: +918197326326, e-mail: drsamskruthii@gmail.com
Source of support: Nil

Conflict of interest: None

Date of received: $02-10-2015$

Date of acceptance: 05-01-2016

Date of publication: April 2016

\section{INTRODUCTION}

Endometriosis is one of the most commonly encountered benign problems in gynecology. Ultrasound and endocrine parameters have been widely accepted as markers of ovarian reserve. Anti-Mullerian hormone $(\mathrm{AMH})$ in conjugation with antral follicle counts (AFCs) is now believed to be an excellent measure for detecting ovarian reserve. ${ }^{1,2}$ Surgical approach has a fundamental role in the management of endometriosis. Laparoscopic cystectomy is currently considered the first-line treatment for endometriomas especially in subfertile patients. The damage to the ovarian reserve inflicted by surgical excision represents the major concern in balancing the reproductive risks and benefits. ${ }^{3}$ The inadvertent removal of the ovarian cortex and loss of follicles during excision causes potential damage to the ovarian reserve. However, the safety of cystectomy is still questioned with respect to damage incurred by the operated ovary. ${ }^{4}$ The loss of normal follicles can be studied by histopathological assessment of the cyst wall. ${ }^{5}$ The diameter of the cyst wall and bilaterality of the cyst influences the postoperative decline in the ovarian reserve. ${ }^{6}$

We undertook this present study so as to evaluate the effect of laparoscopic cystectomy on the ovarian reserve parameters.

Our aim was to analyze the decline in $\mathrm{AMH}$ and AFC postoperatively as compared with preoperative value, with special reference to size and number of endometriomas. So also to correlate the decline in ovarian reserve parameters (AMH and AFC) with follicle loss on histopathological analysis of the cyst wall.

\section{MATERIALS AND METHODS}

\section{Study Design}

This study was a prospective observational study, conducted in the Department of Obstetrics and Gynecology in Tertiary Centre from August 2013 to September 2014 in patients with endometrioma requiring cystectomy. 
Endometrioma, Cystectomy, Size, Number

Ethical committee clearance was obtained (IEC 392/ 2013).

Informed consent was obtained from all the participants of the study.

\section{Inclusion Criteria}

The criterion was all patients with endometrioma undergoing laparoscopic cystectomy.

\section{Exclusion Criteria}

The exclusion criteria were history of previous ovarian surgery, presurgical evidence of premature ovarian insufficiency, clinical or ultrasonographic evidence of ovarian malignancy.

\section{Sample Size Calculation}

Hirakawa et $\mathrm{al}^{7}$ in 2011 published a study on preoperative and postoperative AMH values following cystectomy. They reported that preoperative serum $\mathrm{AMH}$ levels were $3.9 \pm 2.5 \mathrm{ng} / \mathrm{ml}$ (mean $\pm \mathrm{SD})$, which declined to $2.1 \mathrm{ng} / \mathrm{ml}$ following surgery. We hypothesized that a drop in AMH level by $2 \mathrm{ng} / \mathrm{ml}$ would indicate significant decline and hence the sample size was calculated as follows:

Using probability of type 1 error $(\alpha)$ as 0.05 and power of test (1- $\beta$ ) as $0.8(80 \%)$, difference of two means as 2 with expected standard deviation within the group as 2.5 , the sample size obtained was 26 .

Considering the drop-outs, we decided to recruit 50 patients. There were nine drop-outs and hence 41 patients formed part of the study.

\section{Antral Follicle Counts Estimation}

The AFCs were assessed by counting the number of follicles (measuring 2-10 mm) bilaterally using a real time ultrasound, 5.0 to $7.5 \mathrm{MHz}$ endovaginal probe. The parameters were repeated on the second day of their menstrual cycle postoperatively. The ultrasonographic examination was performed by the same examiner on both the occasions to eliminate the interobserver variations. Although the total AFC was assessed, postoperative decline in the AFC was analyzed only in the diseased ovary to eliminate the compensation by unaffected normal ovary.

\section{Anti-Mullerian Hormone Estimation}

Anti-Mullerian hormone was estimated by venous blood sample drawn 1 day prior to surgery and the same repeated 1 month postoperatively. Anti-Mullerian hormone estimation was done in the same laboratory using immune chemiluscence method.
Reference value and interpretation (James and Seifer ${ }^{8}$ )

\begin{tabular}{ll}
\hline AMH value $(\mathrm{ng} / \mathrm{ml})$ & Interpretation (ovarian reserve) \\
\hline$<1$ & Low \\
$1-3.5$ & Mid-range \\
$>3.5$ & Elevated \\
\hline
\end{tabular}

The laparoscopic endometrioma cystectomy was done by stripping technique and the cyst wall obtained was sent for histopathological analysis. The patients in whom complete cystectomy was not possible and in those in whom only cyst aspiration was done due to dense adhesions were excluded from the study. Cautery was not used in all the cases. In case of excessive hemorrhage, the bipolar coagulation was used for hemostasis; however, the duration of usage of cautery was not quantified. Minimal ooze from the endometrioma bed following excision was managed by a local hemostat (gel foam), in no case suturing was done to control the bleeding. The loss of follicles was assessed by histopathological analysis of cyst wall by the same pathologist based on semiquantitative scale. ${ }^{9}$

\section{RESULTS}

This study was a prospective observational study consisting of 41 patients, diagnosed to have endometriomas and managed by laparoscopic cystectomy. As summarized in Table 1, majority of our patients were less than 30 years and more than $85 \%$ of them presented with primary infertility. In the present study, the most common age group was between 26 and 30 years.

The ovarian reserve parameters (AMH and AFC) were studied in 41 patients who underwent laparoscopic cystectomy and there was a statistically significant decline

Table 1: Patient characteristics

\begin{tabular}{lll}
\hline $\begin{array}{l}\text { Characteristics } \\
(n=41)\end{array}$ & Mean $\pm S D /$ percent & Range \\
\hline Age & $29.61 \pm 3.2^{*}$ years & $25-38$ years \\
$\begin{array}{l}\text { Infertility } \\
\quad \text { Primary }\end{array}$ & $36(87.8 \%)^{* *}$ years & \\
$\quad$ Secondary & $5(12.2 \%)$ years & \\
Duration of infertility & $2.48 \pm 1.46^{*}$ years & $1-6$ years \\
Number of the cyst & & \\
$\quad$ Unilateral & $35(85.3 \%)^{* *}$ number $(\%)$ & \\
$\quad$ Bilateral & $6(14.7 \%)$ number $(\%)$ & $3.5-8 \mathrm{~cm}$ \\
$\begin{array}{l}\text { Size of cyst } \\
\text { Preop. AMH }\end{array}$ & $3.5 \pm 1.2^{*} \mathrm{~cm}$ & $0.1-14.92 \mathrm{ng} / \mathrm{ml}$ \\
$\begin{array}{l}\text { Postop. AMH } \\
\text { (1 month later) }\end{array}$ & $2.6 \pm 3.06^{*}$ ng/ml $\pm 1.92^{*}$ ng/ml & $0.1-9 \mathrm{ng} / \mathrm{ml}$ \\
Preop. AFC & $5.17 \pm 1.44^{*}$ (number) & $2-8$ \\
$\quad$ Diseased ovary & & \\
Postop. AFC & $3.61 \pm 1.61^{*}$ (number) & $1-8$ \\
(1 month later) & & \\
$\quad$ Operated ovary & & \\
\hline
\end{tabular}

Values expressed as: *Mean \pm SD; ${ }^{* *}$ Percentage 


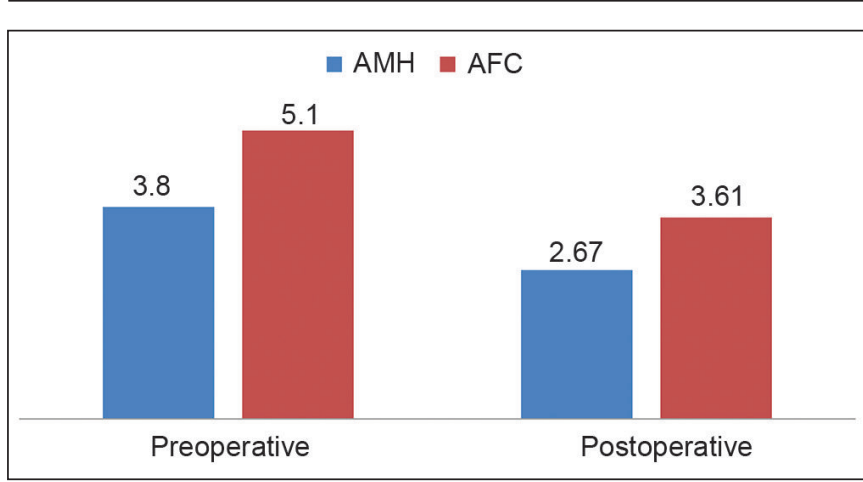

Graph 1: Comparison of ovarian reserve parameters pre- and postcystectomy (statistical test-paired t-test<0.05: significant)

in both AMH and AFC postoperatively $(\mathrm{p}<0.001)$ as compared with the preop value as shown in Graph 1. Thus, it was inferred that cystectomy influences the reserve of ovarian follicles.

Thirty-five patients had unilateral endometrioma and six had bilateral endometriomas. The mean AMH in unilateral and bilateral endometriomas was $3.9 \pm$ 3.17 and $2.9 \pm 1.7 \mathrm{ng} / \mathrm{ml}$ respectively. The mean AFC was $5.2 \pm 1.5$ in unilateral endometriomas and $5 \pm 0.8$ in bilateral endometriomas. There was a drop in $\mathrm{AMH}$ to $2.75 \pm 2.02 \mathrm{ng} / \mathrm{ml}$ and $\mathrm{AFC}$ to $3.5 \pm 1.6$ in unilateral endometriomas and to $2.14 \pm 1.22 \mathrm{ng} / \mathrm{ml}$ and $4 \pm 1.6 \mathrm{in}$ bilateral endometriomas respectively postoperatively. The incidence of unilateral endometriomas was $85 \%$. It was clear from the observation that the mean $\mathrm{AMH}$ and AFC in bilateral endometriomas were lower than that of unilateral endometriomas. As summarized in Table 2, there was a statistically significant decline in ovarian reserve parameters postcystectomy. Bilateral endometriomas with a poor ovarian reserve to begin with had a further decline postcystectomy.

Out of 41 patients studied, 26 had endometrioma of $<5$ $\mathrm{cm}$ and the remaining 15 had endometrioma of $>5 \mathrm{~cm}$ in size. The mean AMH and AFC in patients with smaller cyst $(<5 \mathrm{~cm})$ was $4.5 \pm 3.4 \mathrm{ng} / \mathrm{ml}$ and $5.2 \pm 1.1$ respectively, which showed a decline to $3.9 \pm 2.18 \mathrm{ng} / \mathrm{ml}$ and $3 \pm 1.06$ in the postoperative period. The mean AMH and AFC in patients with larger cyst $(>5 \mathrm{~cm})$ was $2.4 \pm 1.2 \mathrm{ng} / \mathrm{ml}$ and $5.1 \pm 1.6$ respectively, which showed a decline to 1.7 $\pm 0.85 \mathrm{ng} / \mathrm{ml}$ and $3.9 \pm 1.7$ in the postoperative period. As summarized in Table 3, there was a statistically significant decline in the ovarian reserve parameters postoperatively, the decline being more in the smaller cyst $<5 \mathrm{~cm}$.

Histopathological analysis of the cyst wall was done. The loss of follicles was correlated with $\mathrm{AMH}$ and AFC. As summarized in Table 4, 10 (25\%) cysts showed evidence of loss of follicles on histopathological analysis. There was a drop in both AMH and AFC postoperatively. Patients who had loss of follicles (grade 2 and above) on histopathology had relatively more decline in AFC than in $\mathrm{AMH}$. This observation also suggests that the loss of follicles was directly proportional to a drop in AFC that was statistically significant. The decline in AMH was also found to be correlating with AFC and follicle loss on cyst

Table 2: Correlation of ovarian reserve parameters with laterality of the cyst

\begin{tabular}{|c|c|c|c|c|c|c|}
\hline Characters $(n=41)$ & \multicolumn{3}{|c|}{$A M H(n g / m l)(n=41)$} & \multicolumn{3}{|c|}{$A F C(n=41)$} \\
\hline Laterality of cyst & Preoperative & Postoperative & Drop in AMH & Preoperative & Postoperative & Drop in AFC \\
\hline Unilateral $(n=35)$ & $3.9 \pm 3.17$ & $2.75 \pm 2.02$ & 1.15 & $5.2 \pm 1.5$ & $3.5 \pm 1.6$ & 1.7 \\
\hline Bilateral $(n=6)$ & $2.9 \pm 1.7$ & $2.14 \pm 1.22$ & 0.76 & $5.0 \pm 0.8$ & $4.0 \pm 1.6$ & 1 \\
\hline$p$-value & 0.012 & & & 0.02 & & \\
\hline
\end{tabular}

Statistical test-Mauchly's test of sphericity $p<0.05$ : significant

Table 3: Correlation of ovarian reserve parameters with cyst size

\begin{tabular}{|c|c|c|c|c|c|c|}
\hline Characters & \multicolumn{3}{|c|}{$A M H(n g / m l)(n=41)$} & \multicolumn{3}{|c|}{$A F C(n=41)$} \\
\hline Size of cyst & Preoperative & Postoperative & Drop in $\mathrm{AMH}$ & Preoperative & Postoperative & Drop in AFC \\
\hline$<5 \mathrm{~cm}(\mathrm{n}=26)$ & $4.5 \pm 3.4$ & $3.19 \pm 2.18$ & 1.31 & $5.2 \pm 1.1$ & $3.0 \pm 1.06$ & 2.2 \\
\hline$>5 \mathrm{~cm}(\mathrm{n}=15)$ & $2.4 \pm 1.2$ & $1.7 \pm 0.85$ & 0.7 & $5.1 \pm 1.6$ & $3.9 \pm 1.7$ & 1.2 \\
\hline$p$-value & $<0.001$ & & & $<0.001$ & & \\
\hline
\end{tabular}

Statistical test-Mauchly's test of sphericity $p<0.05$ : significant

Table 4: Comparison of ovarian reserve parameters with loss of follicles on cyst wall

\begin{tabular}{|c|c|c|c|c|}
\hline \multirow{2}{*}{$\begin{array}{l}\text { Characters } \\
\text { HPE of cyst wall (Semi-quantitative scale) }\end{array}$} & \multicolumn{2}{|c|}{$A M H(n g / m l)(n=41)$} & \multicolumn{2}{|c|}{$A F C(n=41)$} \\
\hline & Preoperative & Postoperative & Preoperative & Postoperative \\
\hline Grade $0(n=31)$ Complete absence of follicles & $3.9 \pm 3.2$ & $2.6 \pm 2.04$ & $5.32 \pm 1.5$ & $3.84 \pm 1.75$ \\
\hline Grade $1(n=6)$ Only primordial follicles & $2.86 \pm 2.7$ & $2.59 \pm 2.03$ & $4.5 \pm 1.04$ & $2.83 \pm 0.75$ \\
\hline Grade $2(n=1)$ Primordial and primary follicles & $3.7 \pm 2$ & $3.45 \pm 1.9$ & $4 \pm 1.2$ & $3 \pm 1$ \\
\hline Grade $3(n=3)$ Secondary follicles & $3.9 \pm 1.5$ & $2.83 \pm 0.76$ & $5.33 \pm 1.15$ & $3 \pm 1$ \\
\hline p-value & $0.164(\mathrm{NS})^{*}$ & & 0.007 & \\
\hline
\end{tabular}

Statistical test - Mauchly's test of sphericity $p<0.05$ : significant, *NS: Not significant 
wall analysis; however, the observation was not found to be statistically significant.

\section{DISCUSSION}

Endometriosis is considered as the commonest chronic enigmatic disease. Ovarian endometriomas are the commonest form of endometriosis. AMH and AFC are currently believed to be better predictors of ovarian reserve. ${ }^{1,2}$ The gold standard in the treatment of ovarian endometrioma is laparoscopic cystectomy, but the safety of stripping technique of cystectomy is questioned due to its possible deleterious effect on ovarian reserve. ${ }^{3}$ The damage to the ovarian reserve inflicted by surgery presents a major concern in the balance between reproductive risks and benefits. ${ }^{4}$ The accidental removal of ovarian cortex during cystectomy can be assessed by follicular loss on histopathological analysis of the cyst wall. ${ }^{5}$

In the present study, we have analyzed the safety of ovarian cystectomy for endometriomas with respect to number and cyst size, by comparing ovarian reserve parameters (AMH and AFC) before and after cystectomy and also by histopathological analysis of the cyst wall.

As seen in results (Table 1), majority of our patients were less than 30 years of age and more than $85 \%$ of them presented with primary infertility. The present study showed that the ovarian reserve parameters (AMH and AFC) had a declining trend at 1 month postoperatively (Graph 1). This observation was statistically significant with p-value of $<0.001$ applying paired t-test.

Similar to our study, Celik et $\mathrm{al}^{6}$ analyzed 65 patients with unilateral and bilateral endometriomas who underwent laparoscopic cystectomy. In their study, they also compared the mean cyst diameter of $<5$ and $>5 \mathrm{~cm}$ with postoperative decline in AMH. They found that the mean AMH was higher in patients with cyst size $>5$ $\mathrm{cm}$, which was not correlating with our observation. They found that the decline in $\mathrm{AMH}$ was more with bilateral endometriomas and also with cysts $>5 \mathrm{~cm}$. They hypothesized that larger endometriomas may inhibit the transition of follicles from preantral to antral stages and $\mathrm{AMH}$ as we know is secreted from the preantral follicles; hence, AMH may be higher in larger endometriomas.

Celik et $\mathrm{al}^{6}$ showed a significant improvement in AFC at 6 th week and 6th month follow-up postoperatively in their analysis of 65 patients with endometrioma.

A study by Hirakawa et $\mathrm{al}^{7}$ consisting of 38 patients with unilateral and bilateral endometriomas who underwent either laparoscopy or laparotomy cystectomy was analyzed. The serum AMH was evaluated 1 month postoperatively. They concluded that mean $\mathrm{AMH}$ in bilateral endometrioms was lower than that of unilateral endometrioma, which was consistent with our observation (Table 2). Similar to our study, they also demonstrated the postoperative decline in $\mathrm{AMH}$ in both unilateral and bilateral endometriomas.

Chang et $\mathrm{l}^{10}$ analyzed 20 patients (13 endometriomas and 7 nonendometriomas). Anti-Mullerian hormone was measured pre and postoperatively at different intervals (1 week, 1 and 3 months). They found mean AMH of $2.23 \mathrm{ng} / \mathrm{ml}$ preoperatively that reduced to 0.67 and 1.14 at 1-week and 1-month intervals respectively. The results were similar in both endometrioma and nonendometrioma groups. It was interesting to know that Chang et $\mathrm{al}^{10}$ reported an improvement in $\mathrm{AMH}$ at 3 months follow-up (mean AMH of $1.5 \mathrm{ng} / \mathrm{ml}$ ).

In the present study, we showed a decline in AMH 1 month postoperatively. However, subsequent assessment was not possible considering the cost of $\mathrm{AMH}$ assay and loss of patient follow-up.

Results of our study were in agreement with the study by Iwase et $\mathrm{al}^{11}$, who analyzed 65 patients consisting of 29 endometriomas, 21 nonendometriomas, and 15 leiomyomas. All these patients showed a decrease in AMH 1 month postoperatively.

In the present study, we showed that the mean $\mathrm{AMH}$ was low in cysts of $>5 \mathrm{~cm}$. There was a statistically significant decline in $\mathrm{AMH}$ irrespective of cyst size. However, the decline was observed to be more in those patients with endometriomas of $<5 \mathrm{~cm}$ (Table 3 ), which could be because of more damage caused to the surrounding ovarian tissue and also difficulty in identifying the correct plane in the event of removal of a smaller cyst .

For years, a debate has been under way as to the most appropriate surgical technique for endometriomas, comparing the most commonly used techniques: cystectomy, fenestration and bipolar coagulation, or fenestration or laser vaporization of the cyst wall or two or three-step techniques. ${ }^{12}$ These techniques are usually evaluated in terms of their effect on the ovarian reserve.

A cochrane review by Hart et $\mathrm{al}^{3}$ concluded that the excisional surgery for endometriomas had better outcome in terms of recurrence of symptoms, ovarian response to stimulation, and spontaneous pregnancy.

A randomized controlled trial ${ }^{13}$ comparing cystectomy and ablation by bipolar coagulation showed a decline in AFC in both techniques. There was a statistically significant decline in cystectomized ovaries than in coagulated ovaries.

Another randomized controlled trial compared the impact of cystectomy and a three-step laser ablation on ovarian reserve markers, mainly AMH. The study showed a statistically significant decline in surgical trauma in a three-step procedure compared with cystectomy alone. ${ }^{14}$

Said et $\mathrm{al}^{15}$ evaluated ovarian reserve following surgical treatment of unilateral ovarian cyst and concluded that stripping technique of removal of cysts is associated with direct trauma to the remaining ovarian cortex with 
destruction of the follicles. The damage to the ovary was increased in endometriotic cysts, which was statistically insignificant.

A study by Bhat et $\mathrm{al}^{16}$ including 73 patients who underwent laparoscopic cystectomy demonstrated an insignificant decline in the AFC 1 month postoperatively. The observation of this study was in contrast to our study that showed statistically significant decline in AFC of the operated ovary postoperatively. The decline in AFC showed a correlation to loss of follicles on histopathological analysis of the cyst wall (Table 4). Hence, the stripping technique of endometrioma cystectomy can cause ovarian trauma and result in loss of ovarian follicles and lead to decline in ovarian reserve.

\section{Limitations of the Study}

The sample size was small, as only 41 patients formed the part of the study. Ovarian reserve parameters (AMH and AFC) were assessed only once (1 month later) following cystectomy. Hence, long-term effect of cystectomy on the ovarian reserve could not be assessed. Cystectomy only by stripping technique was analyzed in the present study.

\section{CONCLUSION}

The results of this study showed that laparoscopic cystectomy caused further reduction of ovarian reserve as reflected by postoperative assessment of AMH and AFC. Bearing this fact in mind, surgery should be done meticulously. The study also demonstrated that bilateral endometriomas are associated with reduced ovarian reserve measured by serum AMH and ultrasonographic assessment of AFC. The present study also shows that smaller cysts are associated with profound decline in the ovarian reserve. Hence, cystectomy may be safer in larger cysts. From the above study, we also conclude that more than one mechanism is responsible for postoperative decline in ovarian reserve parameters, and though all of our patients had a decline in AMH and AFC during their follow-up period, only a small percentage actually showed loss of follicles by cyst wall analysis.

\section{ACKNOWLEDGMENT}

The authors wish to thank Dr Anuradha CK Rao, Professor, Department of Pathology, Kasturba Medical College, Manipal for her kind support in carrying out the study.

\section{REFERENCES}

1. Ercan CM, Duru NK, Karasahin KE, Coksuer H, Dede M, Baser I. Ultrasonographic evaluation and antimullerian hormone levels after laparoscopic stripping of unilateral endometriomas. Eur J Obstet Gynecol Reprod Biol 2011 Oct;158(2):280-284.
2. Scheffer GJ, BroekmansFJ, Bansci LF, Habbema JD, Looman CW, Te Velde ER. Quantitative transvaginal two and three dimensional sonography of the ovaries: reproducibility of antral follicle counts. Ultrasound Obstet Gynecol 2002 Sep;20(3):270-275.

3. Hart RJ, Hickey M, Maouris P, Buckett W. Excisional surgery versus ablative surgery for ovarian endometriota. Cochrane Database Syst Rev 2008 Apr16;(2):CD004992.

4. Li CZ, Liu B, Wen ZQ, Sun Q. The impact of electrocoagulation on ovarian reserve after laparoscopic excision of ovarian cysts: a prospective clinical study of 191 patients. Fertil Steril 2009 Oct;92(4):1428-1435.

5. Hnsen KR, Hodnett GM, Knowlton N, Craig LB. Correlation of ovarian reserve tests with histologically determined primordial follicle number. Fertil Steril 2011 Jan;95(1):170-175.

6. Celik GH, Dogan E, Okyay E, Ulukus C, Saatli B. Effect of laparoscopic excision of endometriomas on ovarian reserve: serial changes in the serum antimullerian hormone levels. Fertil Steril 2012 Jun;97(6):1472-1478.

7. Hirakawa W, Iwase A, Goto M, Takikawa S, Nagatomo $Y$, Nakahara T, Bayasula B, Nakamura T, Manabe S, Kikkawa F. The postoperative decline in serum anti-mullerian hormone correlates with the bilaterality and severity of endometriosis. Hum Reprod 2011 Apr;26(4):904-910.

8. James PT, Seifer DB. Why we may abandon basal folliclestimulating hormone testing: a sea change in determining ovarian reserve using antimullerian hormone. Fertil Steril 2013 Jun;99(7):1825-1830.

9. American College of Obstetricians and Gynecologists. Medical management of endometriosis. ACOG Practice Bulletin No. 11, Obstet Gynecol 1999 Dec;94(16):1-14.

10. Chang HJ, Han SH, Lee JR, Jee BC, Lee BI, Suh CS, Kim SH. Impact of laparoscopic cystectomy on ovarian reserve: serial changes of serum antimullerian hormone levels. Fertil Steril 2010 Jun;94(1):343-349.

11. Iwase A, Hirakata W, Goto M, Takikawa S, Nagamoto $Y$, Nakamura T, Manabe S, Kikkawa F. Serum anti-mullerian hormone level is useful marker for evaluating the impact of laparoscopic cystectomy on ovarian reserve. Fertil Steril 2010 Dec;94(7):2846-2849.

12. Tsolakidis D, Pados G, Vavilis D, Athanatos D, Tsalikis T, Giannakou A, Tarlatzis BC. The impact on ovarian reserve after laparoscopic ovarian cystectomy versus three stage management in patients with endometriomas: a prospective randomized study. Fertil Steril 2010 Jun;94(1):71-77.

13. Var T, Batioglu S, Tonguc E, Kahyaoglu I. The effect of laparoscopic ovarian cystectomy versus coagulation in bilateral endometriomas on ovarian reserve as determined by antral follicle count and ovarian volume: a prospective randomized study. Fertil Steril 2011 Jun;95(7):2247-2250.

14. Pados G, Tsolakidis D, Athanatos D, Tarlatzis B. Sonographic changes after laparoscopic cystectomy compared with threestage management in patients with ovarian endometriomas: a prospective randomized study. Hum Reprod 2010 Mar;25(3): 672-677.

15. Said TH, El Sibai F, Rocca M, Kholif A, Ahmed A, Soliman A. Evaluation of ovarian reserve after surgical treatment of unilateral benign ovarian cyst. Fertil Steril. 2009;91,(Suppl 1): S20.

16. Bhat RG, Dhulkhed S, Ramachandran A, Bhaktha R, Vasudeva A, Kumar P, Rao AC. Laparoscopic cystectomy of endometrioma: good surgical technique does not adversely affect ovarian reserve. J Human Reprod Sci 2014 Apr;7(2):125-129. 